\section{GW23-e2592 THE STUDY OF APOPTOSIS MECHANISM IN THE PROCESS OF RECOMBINANT INTERLEUKIN-12 TREAT THE MICE WITH VIRAL MYOCARDITIS}

doi:10.1136/heartjnl-2012-302920a.198

Yu Xia, Han Li-zhi, Sun Jing-hui, piao Jin-hua, piao Jin-hua. The First Clincal Hospital of Jilin University, Jilin Changchun130021, China

Objectives Through studying the NK cell activity, the pathological changes of myocardial cells and the changes of apoptosis in the process of recombinant interleukin -12 treat the mice with viral myocarditis, to explore the therapeutic effect and mechanism of apoptosis in the process of recombinant interleukin -12 treat the viral diseases, to further reveal the treatment effect and therapeutic mechanism of recombinant interleukin-12 to viral diseases, and to make a theoretical foundation for the clinical use of recombinant interleukin -12 .

Methods A total of $90 \mathrm{BALB} / \mathrm{C}$ inbreeding male mice weight 14-18 g were randomly divided into six groups, each group has 15 mice. They are blank control group, the virus control group, drug control group, rmIL-12 small dose treatment group, medium dose treatment group, high dose treatment group. Each mouse was injected Coxsackie B3 $0.2 \mathrm{ml} / \mathrm{d}$ for three consecutive days by intraperitoneal injection, in addition to except the mouse in blank control group. After inoculating $4 \mathrm{~h}$, in the blank control group and the virus control group, each mouse was inoculated $0.9 \%$ saline $0.1 \mathrm{ml} / \mathrm{d}$ by intraperitoneal injection, in the drug control group, each mouse was inoculated interferon-of $\gamma 400 \mathrm{IU} /$ $\mathrm{d}$, rmIL-12 small-dose, medium-dose and high-dose treatment group, the mouse in each group were injected recombinant interleukin-12 respectively $1 \mathrm{ng} / \mathrm{d}, 10 \mathrm{ng} / \mathrm{d}, 100 \mathrm{ng} / \mathrm{d}$ for five consecutive days. The time of injecting virus is the zeroth day. At the fifth day, we take 10 mice from each group, than killed them by cervical dislocation. We removed the spleens for testing the NK cell activity, under sterile conditions. We removed the hearts, than make heart specimens. We soaked the heart in $10 \%$ formalin solution and marked them for the test of heart pathology and apoptosis. NK cell activity was measured by MTT assay. Apoptosis was detected by TUNEL and pathological scores. Cardiac muscle pathology was detected by HE staining. The statistical data was expressed as mean \pm SD $(\square \pm S)$, The data were analysed by SPSS17.0 statistical software analysis system. Groups were analysed using analysis of variance. $p<0.05$ was considered as statistically significant.
Results In addition to the blank control group, each group can be detected the apoptosis of myocardial cells. The pathological scores of apoptotic cells are that, high dose treatment group is $29.18 \%$, medium dose treatment group is $20.97 \%$, small dose treatment group is $12.03 \%$, drug control group is $10.51 \%$, virus control group is $15.63 \%$. The largest number of apoptotic cells is the high dose treatment group, followed by the middle dose treatment group, and the low dose treatment group and the virus control group is less than the above two groups. The NK cell activity in each group is that, blank control group is $29.58 \pm 4.56 \%$, virus control group is $46.99 \pm 6.31 \%$, drug control group is $34.54 \pm 3.98 \%$, small dose treatment group is $42.14 \pm 4.02 \%$, medium dose treatment group is $54.55 \pm 7.17 \%$, high dose treatment group $57.06 \pm 4.55 \%$. There are statistically significant differences in different groups about NK cell activity, $\mathrm{F}=34.98, \mathrm{p}<0.01$. There are significant differences in medium-dose group, virus control group and the drug control group $\mathrm{p}<0.05)$. There are not significant differences between drug control group and small dose treatment group. Myocardial pathological changes (the degeneration and necrosisis of myocardial cell) are lighter in medium-dose treatment group than the other treatment groups. And, the virus control group and the high dose treatment group are most severe than other groups. The blank control group has not the degeneration and necrosisis.

Conclusions In middle-dose treatment group, the number of myocardial cell degeneration and necrosis is least, indicating recombinant interleukin -12 have therapeutic action to viral myocarditis. The number of apoptotic myocardial cells increased with therapeutic dose of recombinant interleukin -12 , and moderate apoptosis have an active role to clear virus-infected cells. Which indicating apoptosis is one of the treatment mechanism of recombinant interleukin-12. 